\title{
Suppression of intestinal carcinogenesis in Apc-mutant mice by limonin
}

\author{
Satomi Shimizu, ${ }^{1,2}$ Shingo Miyamoto, ${ }^{1}$ Gen Fujii,, ${ }^{1}$ Ruri Nakanishi, ${ }^{1}$ Wakana Onuma, ${ }^{1}$ Yoshihiko Ozaki, ${ }^{3}$ \\ Kyoko Fujimoto, ${ }^{4}$ Tomohiro Yano ${ }^{2}$ and Michihiro Mutoh ${ }^{1, *}$ \\ ${ }^{1}$ Epidemiology and Prevention Division, Research Center for Cancer Prevention and Screening, National Cancer Center, \\ 5-1-1 Tsukiji, Chuo-ku, Tokyo 104-0045, Japan \\ ${ }^{2}$ Graduate School of Life Sciences, Toyo University, 1-1-1 Izumino, Itakura-machi, Oga-gun, Gunma 374-0193, Japan \\ ${ }^{3}$ Faculty of Biology-Oriented Science and Technology, Kinki University, 930 Nishimitani, Kinokawa City, Wakayama 649-6493, Japan \\ ${ }^{4}$ Division of Molecular Biology, Nagasaki International University, 2825-7 Huis Ten Bosch, Sasebo, Nagasaki 859 3298, Japan
}

\begin{abstract}
Limonoids in citrus fruits are known to possess multiple biological functions, such as anti-proliferative functions in human cancer cell lines. Therefore, we aimed to investigate the suppressive effect of limonin on intestinal polyp development in Apc-mutant Min mice. Five-week-old female Min mice were fed a basal diet or a diet containing 250 or 500 ppm limonin for 8 weeks. The total number of polyps in mice treated with $\mathbf{5 0 0} \mathrm{ppm}$ limonin decreased to $\mathbf{7 4 \%}$ of the untreated control value. Neoplastic cell proliferation in the polyp parts was assessed by counting PCNA positive cells, and a tendency of reduction was obtained by limonin treatment. Moreover, expression levels of c-Myc and MCP-1 mRNA in the polyp part were reduced by administration of limonin. We finally confirmed the effects of limonin on $\beta$-catenin signaling, and found limonin significantly inhibited T-cell factor/lymphocyte enhancer factor-dependent transcriptional activity in a dose-dependent manner in the Caco-2 human colon cancer cell line. Our results suggest that limonin might be a candidate chemopreventive agent against intestinal carcinogenesis.
\end{abstract}

Key Words: limonin, Min mice, colon cancer, c-Myc, Tcf/Lef

$\mathrm{C}$ olorectal cancer is the third most common cancer in the world. Nearly 1.4 million new cases occurred in 2012 , and this is expected to increase to 2.4 million annually by 2035 worldwide.(1) Despite extensive efforts to develop many anti-cancer drugs, mortality of colorectal cancer is still high. Therefore, a new strategy for controlling development of cancer is in great demand. ${ }^{(2)}$ One approach is the use of functional agents in foods or plants as supplements or adjuvant agents in chemotherapy. ${ }^{(3,4)}$ The other approach is chemoprevention for the inhibition of colorectal cancer development. ${ }^{(3,4)}$ Since epidemiological studies suggest that a high intake of fruit is associated with a reduced risk of chronic disease, including cancer, ${ }^{(5)}$ using functional agents in citrus fruits seems to be an attractive approach for both methods.

Citrus fruits are good resources for a variety of constituents important to human nutrition, such as vitamin C, folic acid, potassium, flavonoids, pectin, and dietary fiber. Citrus also contains highly oxygenated triterpenoids (limonoids), which contain a furan ring attached to the D-ring at C-17 as well as an epoxide group at $\mathrm{C}-14$ and $\mathrm{C}-15$, and oxygen containing functional groups at C-3, C-4, C-7, C-16 and C-17. Limonoids were firstly identified as components contributing to bitterness in citrus juices. ${ }^{(6)} \mathrm{Re}-$ cently, it is has been revealed that limonoids possess multiple biological functions in vitro and in vivo. ${ }^{(7)}$ Notably, limonin, the most abundant limonoid in citrus fruits, showed anti-proliferative efficacy in several human cancer cell lines ${ }^{(8-10)}$ and a suppressive effect on the development of aberrant crypt foci (ACF) in AOM- treated rats. ${ }^{(11)}$

Although several reports have shown that limonin exhibits anticancer effects in vitro and suppressive effects on the formation of preneoplastic lesions in vivo, the effects of limonin on intestinal carcinogenesis have not been addressed. In this study, we investigated the impact of limonin on intestinal polyp formation in Apc-mutant Min mice, an animal model of human familial adenomatous polyposis that develops numerous polyps in the intestinal tract. We also evaluated the effects of limonin on the transcriptional activity of the Wnt signaling pathway, which plays an important role in the proliferation of cancer cells, in a human colon cancer cell line.

\section{Materials and Methods}

Cell cultures and chemicals. Caco- 2 cells, a human colon adenocarcinoma cell line, were purchased from Sumitomo Dainippon Pharma Co., Ltd. (Osaka, Japan). The cells were maintained in DMEM medium supplemented with $10 \%$ heatinactivated fetal bovine serum (FBS; Hyclone Laboratories Inc., Logan, UT), MEM Non-Essential Amino Acids Solution (Nacalai Tesque, Inc., Japan) and antibiotics $(100 \mu \mathrm{g} / \mathrm{ml}$ streptomycin and $100 \mathrm{U} / \mathrm{ml}$ penicillin) at $37^{\circ} \mathrm{C}$ in $5 \% \mathrm{CO}_{2}$. Limonin for animal study was isolated from grapefruit seed, and kindly provided by Dr. Shin Hasegawa (USDA, ARS, WRRC, retired). Limonin for cell culture study was purchased from Wako (Osaka, Japan).

Animals. Female C57BL/6-Apc $c^{\mathrm{Min} /+}$ mice (Min mice) were purchased from The Jackson Laboratory (Bar Harbor, ME). Mice were housed per plastic cage with sterilized softwood chips as bedding in a barrier-sustained animal room at $24 \pm 2{ }^{\circ} \mathrm{C}$ and $55 \%$ humidity on a $12 \mathrm{~h} \mathrm{light/dark} \mathrm{cycle.} \mathrm{Limonin} \mathrm{was} \mathrm{well} \mathrm{mixed} \mathrm{at} \mathrm{a}$ concentration of 250 or $500 \mathrm{ppm}$ in AIN-76A powdered basal diet (CLEA Japan, Tokyo, Japan).

Protocol for animal experiments. Ten female Min mice at 5 weeks of age were given 250 or 500 ppm limonin for 8 weeks. The animals in each cage were all in the same treatment group. Food and water were available ad libitum. The animals were observed daily for clinical signs and mortality. Body weight and food consumption were measured weekly. The intestinal tract was removed and separated into the small intestine, cecum and colon. The small intestine was divided into the proximal segment $(4 \mathrm{~cm}$ in length) from the stomach and then the proximal (middle) and distal halves of the remaining part. Polyps in the proximal segments were counted immediately, and all polyps in the proximal

*To whom correspondence should be addressed. E-mail: mimutoh@ncc.go.jp 
Table 1. Number of intestinal polyps/mouse in Min mice

\begin{tabular}{ccccccc}
\hline \multirow{2}{*}{ Limonin $(\mathrm{ppm})$} & \multirow{2}{*}{ No. of mice } & \multicolumn{3}{c}{ Small intestine } & \multirow{2}{*}{ Colon } & \multirow{2}{*}{ Total } \\
\cline { 3 - 6 } & & Proximal & Middle & Distal & & $35.2 \pm 9.4$ \\
250 & 10 & $4.9 \pm 2.1$ & $9.7 \pm 3.2$ & $18.0 \pm 7.4$ & $0.0 \pm 0.0$ & $29.2 \pm 5.4$ \\
500 & 10 & $3.8 \pm 2.0$ & $8.9 \pm 2.6$ & $16.3 \pm 6.3$ & $0.2 \pm 0.4$ & $25.6 \pm 5.2^{*}$ \\
\hline
\end{tabular}

Data are mean \pm SD. *Significantly different from the control untreated group at $p<0.05$.

segment were picked up under a stereoscopic microscope and the remaining intestinal mucosa (non-polyp part) was removed by scraping, and then both stored at $-80^{\circ} \mathrm{C}$ for further analysis. Other segments were opened longitudinally and fixed flat between sheets of filter paper in $10 \%$ buffered formalin. The numbers and sizes of polyps and their distributions in the intestine were assessed with a stereoscopic microscope after fixation. The experiments were performed according to the "Guidelines for Animal Experiments in the National Cancer Center" and were approved by the Institutional Ethics Review Committee for Animal Experimentation in the National Cancer Center.

Immunohistochemical analysis. Small intestines (middle segment) were sliced and processed to sections stained with hematoxylin and eosin (H\&E), after polyp count. Sections in the middle parts of small intestines were also stained immunohistochemically with antibodies against proliferation cell nuclear antigen (PCNA; DAKO, Carpinteria, CA) and $\beta$-catenin (BD Transduction Lab., Franklin Lakes, NJ) used at $100 \times$ and $200 \times$ dilution, respectively. The number of PCNA positive cells and $\beta$ catenin positive cells were counted from three different arbitrarily selected areas in a polyp $(n=4)$. As the secondary $\mathrm{Ab}$, biotinylated horse anti-mouse IgG affinity purified was employed at $200 \times$ dilution. Staining was performed using avidin-biotin reagents (Vectastain ABC reagents; Vector Laboratories), 3,3'-diaminobenzidine and hydrogen peroxide, and the sections were counterstained with hematoxylin to facilitate orientation. As a negative control, consecutive sections were immunostained without exposure to the primary $\mathrm{Ab}$. Both the index of cell growth and nuclear $\beta$-catenin accumulation were calculated by the formula; positive cells/ total cells in selected area.

Quantitative real-time polymerase chain reaction (PCR) analysis. Total RNA was isolated using TRIzol Reagent (Invitrogen; Grand Island, NY), treated with DNase (Invitrogen), and $1 \mu \mathrm{g}$ aliquots in a final volume of $20 \mu \mathrm{l}$ were used for synthesis of cDNA using a High Capacity cDNA Reverse Transcription kit (Applied Biosystems, Foster City, CA). Real-time PCR was carried out using Fast Start Universal SYBR Green Mix (Roche Diagnostics, Mannheim, Germany) according to the manufacturer's instructions. Primers for COX-2 (5'-GTG CCA ATT GCT GTA CAA GC and 5'-TAC AGC TCA GTT GAA CGC CT), c-Myc (5'-ATG CCC CTC AAC GTG AAC TTC and 5'-GTC GCA GAT GAA ATA GGG CTG), MCP-1 (5'-TAA AAA CCT GGA TCG GAA CCA AA and 5'-GCA TTA GCT TCA GAT TTA CGG GT), VCAM-1 (5'-AGT TGG GGA TTC GGT TGT TCT and 5'-CCC CTC ATT CCT TAC CAC CC), and glyceraldehyde-3-phosphate dehydrogenase (GAPDH) (5'-TGT CAG CAA TGC ATC CTG CA and 5'-TTA CTC CTT GGA GGC CAT GT) were employed. To assess the specificity of each primer set, amplicons generated from the PCR reaction were analyzed for melting curves.

Luciferase assay for Tcf/Lef-dependent transcriptional activity. To measure T-cell factor/lymphoid-enhancer factor (Tcf/Lef)-dependent transcriptional activity, Caco-2 colon cancer cells were seeded into 12 -well plates $\left(1.0 \times 10^{5}\right.$ cells/well). After $24 \mathrm{~h}$ incubation, cells were transiently transfected with $1 \mu \mathrm{g} / \mathrm{well}$ of either TOPflash or FOPflash reporter plasmid (Millipore, Billerica, MA), and pGL4.73 [hRluc/SV40] (Promega Co., Madison, WI) control plasmid using Polyethylenimine "MAX"
Transfection Reagent (Polysciences Inc., Warrington, PA) according to the instructions provided by the manufacturer, and cultured for $24 \mathrm{~h}$. Experiments for the effects of test compounds on Tcf/Lef transcriptional activity plasmid-transfected Caco-2 cells were cultured with $100,200,400$, and $800 \mu \mathrm{M}$ limonin. Finally, firefly luciferase and renilla luciferase activities were determined by Luciferase Assay Systems and Renilla Luciferase Assay Systems (Promega), respectively. Basal luciferase activity of untreated cells was set as 1.0 . The percentage luciferase activity with each treatment was calculated from data for triplicate wells. The value was normalized by renilla luciferase activity. All experiments were repeated at least three times with nearly identical results. Data are expressed as means $\pm \mathrm{SD}(n=5)$. Tcf/ Lef transcriptional activity was normalized for the transfection rate calculated by SV40 promoter driven renilla luciferase.

Statistical analysis. All the results are expressed as mean \pm $\mathrm{SD}$ values, with statistical analysis using the Bonferroni $z$ test, except for mRNA examination in the Min mice intestinal mucosa (Student $t$ test) and immunohistochemical analysis (Student $t$ test). Differences were considered to be statistically significant at $p<0.05$.

\section{Results}

Suppression of intestinal polyp formation in Min mice by limonin. Administration of 250 or $500 \mathrm{ppm}$ limonin to Min mice for 8 weeks did not affect body weight, food intake or clinical signs (activity, glossy coat) throughout the experimental period. Average daily food intake did not significantly differ among the 0,250 and $500 \mathrm{ppm}$ group of Min mice, respectively. In addition, there were no changes observed in any organ weights that might have been attributable to toxicity. Table 1 summarizes data for the number and distribution of intestinal polyps in the basal diet and limonin-treated groups. Almost all polyps developed in the small intestine, with only a few in the colon. The total number of polyps treated with $500 \mathrm{ppm}$ limonin was decreased to $74 \%$ of the untreated control value. Reduction of polyps was observed in the distal part, and was by $73 \%(p<0.05$ vs $0 \mathrm{ppm})$. In the other parts of the small intestine and colon, treatment with limonin lowered the number of polyps without significant difference. Fig. 1 shows the size distribution of intestinal polyps in the basal diet and limonin-treated groups. The maximal number of polyps was observed in the size range between 0.5 and $2.0 \mathrm{~mm}$ in diameter. Administration of limonin significantly reduced the numbers of polyps sized $<0.5 \mathrm{~mm}$ in diameter.

Decrease of an index of cell growth and nuclear $\beta$-catenin accumulation in intestinal polyps by limonin. To investigate the effect of limonin treatment on epithelial cell proliferation, intestinal polyps in Min mice were immunohistochemically stained with anti-proliferation cell nuclear antigen (PCNA) antibody. As shown in Fig. 2, an index of cell growth (PCNA positive cells/total cells in polyp) revealed that administration of $500 \mathrm{ppm}$ limonin had a tendency to suppress cell proliferation in intestinal polyps in Min mice. In addition, the number of cells in polyps that stained for $\beta$-catenin in the nucleus was counted. No significant differences of nuclear $\beta$-catenin accumulation between the control and limonin-treated groups were observed. 


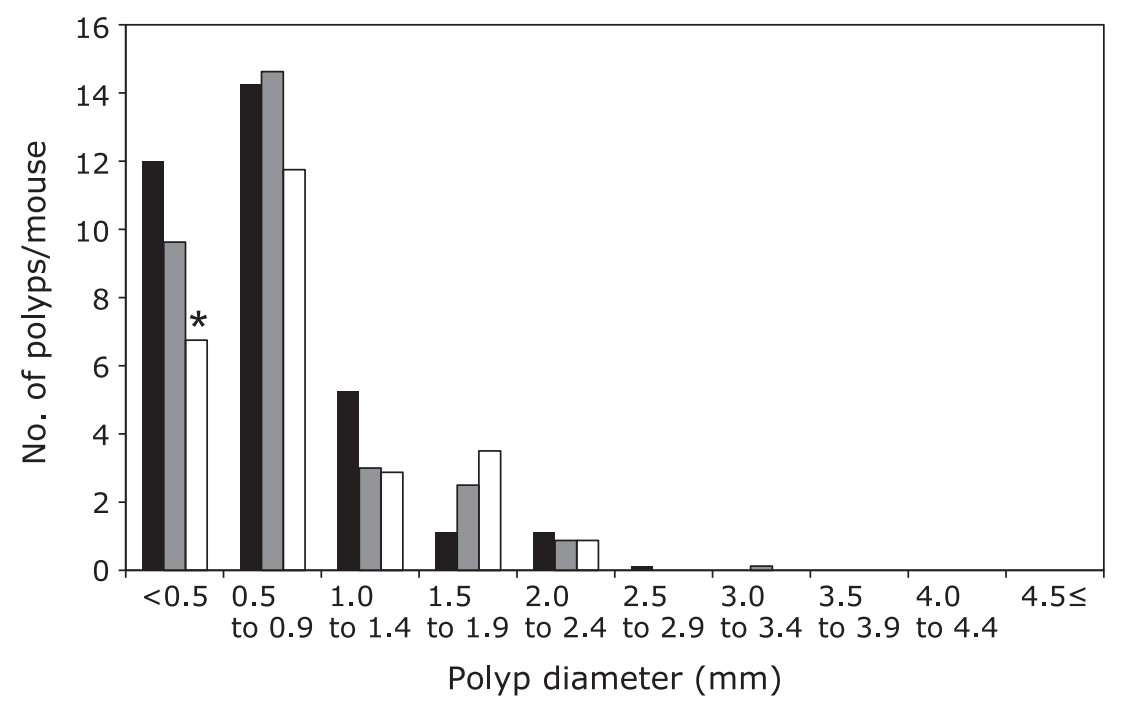

Fig. 1. Effects of limonin on the size distribution of intestinal polyps in Min mice. Min mice were fed a basal diet (black filled box), 250 ppm (gray filled box) and 500 ppm (open box) limonin containing diet for 8 weeks. The number of polyps per mouse in each size class is given as a mean. ${ }^{*} p<0.05$.
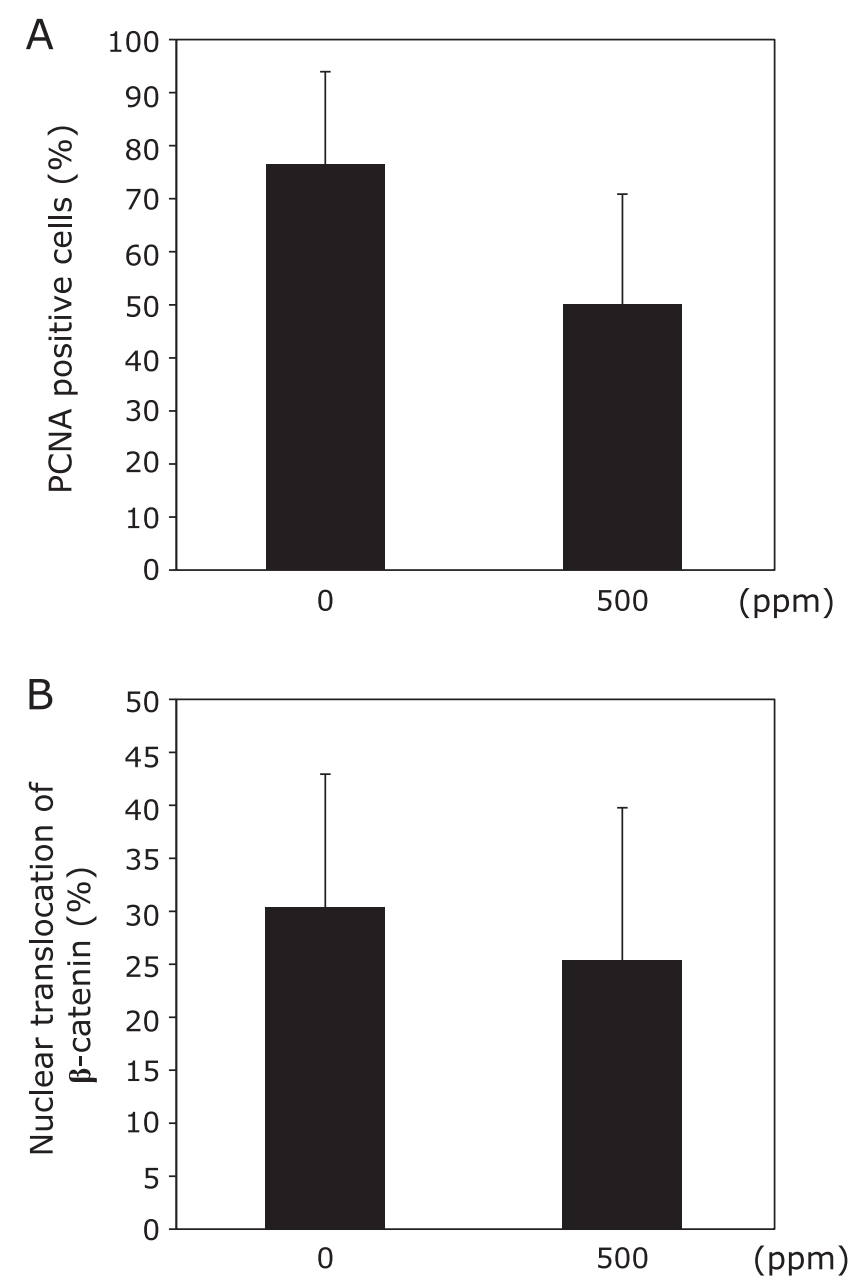

Fig. 2. Change of ratio of PCNA and nuclear $\beta$-catenin accumulation in the intestinal polyp by limonin. Immunohistochemical staining for PCNA and $\beta$-catenin in intestinal polyp from Min mice fed a basal diet or limonin containing diet. Calculations of the ratio for PCNA (A), nuclear $\beta$-catenin accumulation (B) are described in Materials and Methods section. Bars indicate SD.
Suppression of mRNA levels of inflammation- or proliferation-related factor in intestinal polyp of Min mice by limonin. To clarify the mechanisms that suppress intestinal polyp formation by limonin, inflammation-related or proliferation -related factor expression levels in intestinal non-polyp and polyp parts were investigated (Fig. 3). Real-time PCR revealed that treatment with $500 \mathrm{ppm}$ limonin for 8 weeks weakly but significantly suppressed c-Myc, MCP-1 and VCAM-1 mRNA levels in the intestinal polyp parts to $67 \%(p<0.001), 78 \%$ $(p<0.05)$ and $81 \%(p<0.05)$ of that of the untreated value, respectively. COX-2 mRNA expression levels in non-polyp parts and polyp parts of small intestines were not suppressed by limonin treatment.

Inhibition of Tcf/Lef-dependent transcriptional activity by limonin in human colon cancer cells. Although the ratio of nuclear $\beta$-catenin accumulation was low, we confirmed whether the $\beta$-catenin signaling pathway is responsible for the polyp suppressive effect of limonin or not. We measured Tcf/Lefdependent transcriptional activity using a luciferase reporter gene assay. Limonin significantly inhibited Tcf/Lef-dependent transcriptional activity in a dose-dependent manner in the Caco-2 cells (Fig. 4). A dose of $400 \mu \mathrm{M}$ limonin was enough to decrease Tcf/Lef-dependent transcriptional activity to a half. The maximum dose of DMSO in the medium used in this assay did not influence the activity of Tcf/Lef-dependent transcriptional activity (data not shown).

\section{Discussion}

In the present study, we showed the suppressive effect of limonin on intestinal tumor formation in $A p c$-mutant Min mice for the first time. Notably, limonin treatment mainly reduced the number of relatively small polyps $(<0.5 \mathrm{~mm}$ in diameter $)$. Moreover, limonin may have the potential to inhibit the transcriptional activity of $\beta$-catenin, which may partly explain the suppressive effects of limonin on intestinal polyp formation.

In the early stage of intestinal tumorigenesis, activation of the Wnt signaling pathway following loss or mutation of $A p c$ is the key step for the initiation of polyp formation, not only in $A p c$ mutant Min mice but also in humans. ${ }^{(12,13)}$ Activation of the Wnt signaling pathway plays a critical role during normal development as well as during tumorigenesis in animals. ${ }^{(14)} A p c$ protein is one of the main components of the Wnt signaling pathway and normally 

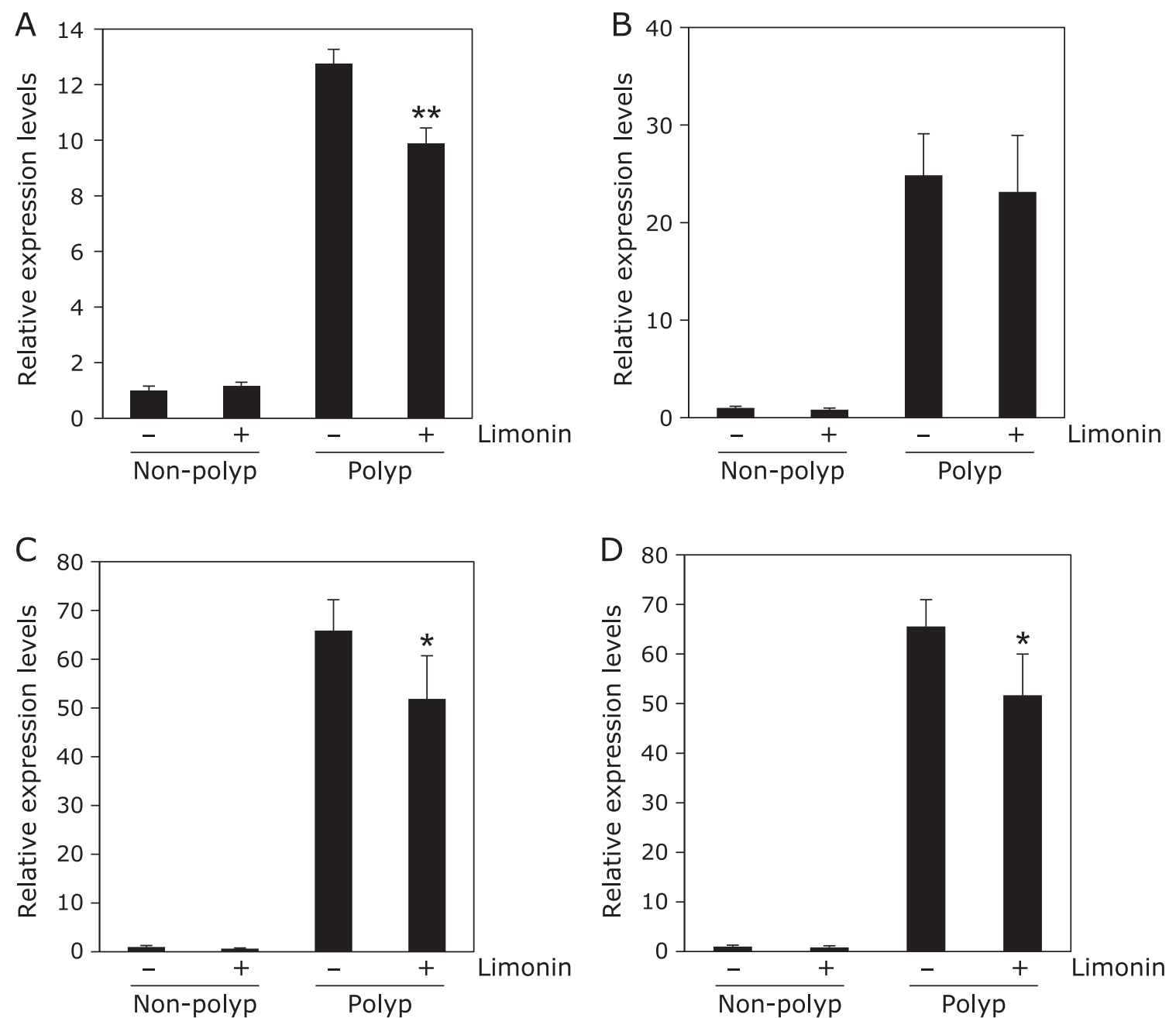

Fig. 3. Changes of inflammation- or proliferation-related factors in intestinal non-polyp and/or polyp parts of Min mice. Quantitative real-time PCR analysis were performed to determine c-Myc (A), COX-2 (B), MCP-1 (C), VCAM-1 (D) mRNA expression levels in the non-polyp or polyp parts of Min mice with or without $500 \mathrm{ppm}$ limonin. Data are normalized with GAPDH expression level. Each expression levels in non-polyp parts of untreated group are set as 1 . Data are mean $\pm \mathrm{SD}, n=6 .{ }^{* *} p<0.001,{ }^{*} p<0.05$ vs $0 \mathrm{ppm}$.

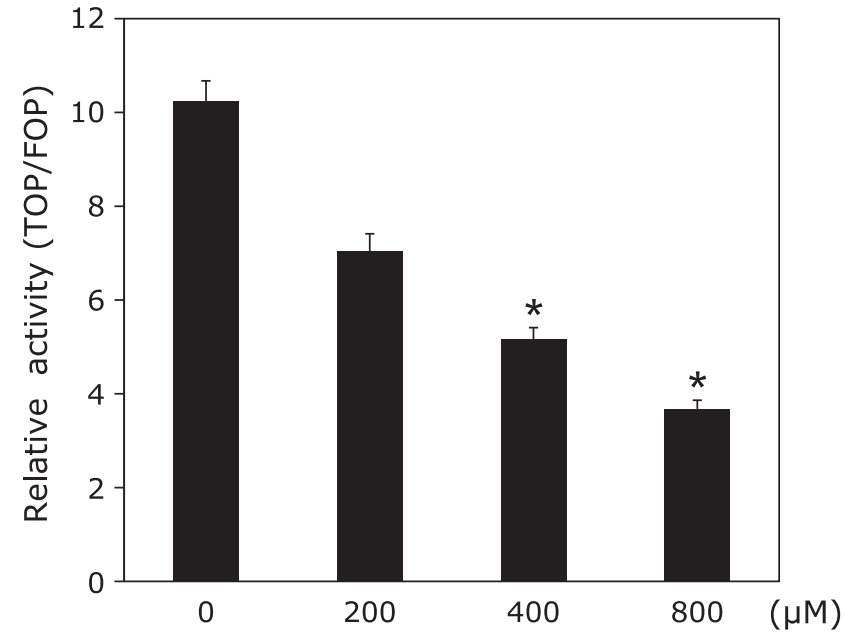

Fig. 4. Effects of limonin treatment on Tcf/Lef-dependent transcriptional activity in human colon cancer cells. Caco-2 cells were cultured in medium containing with the indicated dose of limonin. Relative Tcf/Lefdependent transcriptional activities are plotted as the ratio of the un-stimulated control value. Data are mean $\pm S D, n=6 .{ }^{*} p<0.001$ vs $0 \mu \mathrm{M}$. Similar results were obtained from three separate experiments. regulates pathway activation by binding to and degrading $\beta$ catenin protein. ${ }^{(15)} \beta$-catenin protein is normally found in the cell membrane but, as a result of mutation or loss of $A p c$ and the activation of Wnt signaling with ligand stimulation, it translocates into the nucleus. Indeed, the accumulation of $\beta$-catenin in the cytosol and nucleus is a characteristic phenotype of the intestinal polyp in Min mice. After translocation, $\beta$-catenin protein binds to Tcf/Lef transcription factor and transactivates target genes involved in cell proliferation, such as c-Myc. ${ }^{(16)}$

In our experiment, percentages of PCNA positive proliferative cells and nuclear $\beta$-catenin positive cells were about 80 and $30 \%$ within intestinal tumors of Min mice in the control group, respectively. Although there were no significant differences, $500 \mathrm{ppm}$ limonin administration reduced both percentages in the polyp part compared to the control group. These results suggest that limonin administration reduced tumor numbers partly through the suppression of tumor cell proliferation.

To clarify the underlying mechanisms of suppressive effects of limonin on tumor cell proliferation, we investigated the expression levels of $\beta$-catenin target genes, such as c-Myc, Cyclin D1, MCP1 and VCAM-1 in the polyp parts ${ }^{(17-20)}$ Expression levels of c-Myc and MCP-1, but not Cyclin D1 (data not shown), were significantly reduced within polyps from limonin treated mice. Interest- 
ingly, deletion of c-Myc, ${ }^{(21)}$ but not Cyclin D1, fully rescued early stage $A p c$-loss driven tumorigenesis in the small intestine of mice. Sansom et al. ${ }^{(22)}$ concluded that Cyclin D1 is not an immediate target of $\beta$-catenin and it may require other factors to initiate its expression in a delayed time frame. Since limonin significantly inhibited Tcf/Lef-dependent transcriptional activity in human colon cancer cells, different regulations of transactivation of c-Myc and Cyclin D1 may explain the various effects of limonin on expression levels of c-Myc and Cyclin D1 in the polyp parts of Min mice.

Limonin is known as a primary source of undesirable bitterness in the juice of citrus fruits and $6 \mathrm{ppm}$ is the objectionable level for humans. ${ }^{(6)}$ Taking into consideration that limonin glucoside is involved in commercially available citrus juice at almost $180 \mathrm{ppm},{ }^{(23)}$ and is involved in Satsuma mandarin juice at around $130 \mathrm{ppm},{ }^{(24)}$ the amount of $500 \mathrm{ppm}$ will be achieved by taking limonin as a supplement.

From the present results, we can conclude that limonin may have suppressive effects on intestinal polyp formation partly through the inhibition of the transcriptional activity of $\beta$-catenin. Limonin could be a candidate chemopreventive agent for intestinal cancer.

\section{References}

1 Siegel R, Naishadham D, Jemal A. Cancer statistics, 2012. CA Cancer J Clin 2012; 62: 10-29.

2 Sporn MB, Suh N. Chemoprevention: an essential approach to controlling cancer. Nat Rev Cancer 2002; 2: 537-543.

3 Ferguson LR, Schlothauer RC. The potential role of nutritional genomics tools in validating high health foods for cancer control: broccoli as example. Mol Nutr Food Res 2012; 56: 126-146.

4 Temraz S, Mukherji D, Shamseddine A. Potential targets for colorectal cancer prevention. Int J Mol Sci 2013; 14: 17279-17303.

5 Riboli E, Norat T. Epidemiologic evidence of the protective effect of fruit and vegetables on cancer risk. Am J Clin Nutr 2003; 78: 559S-569S.

6 Manners GD. Citrus limonoids: analysis, bioactivity, and biomedical prospects. J Agric Food Chem 2007; 55: 8285-8294.

7 Zhang Y, Wang JS, Wang XB, et al. Limonoids from the fruits of Aphanamixis polystachya (Meliaceae) and their biological activities. J Agric Food Chem 2013; 61: 2171-2182.

8 Patil JR, Chidambara Murthy KN, Jayaprakasha GK, Chetti MB, Patil BS. Bioactive compounds from Mexican lime (Citrus aurantifolia) juice induce apoptosis in human pancreatic cells. J Agric Food Chem 2009; 57: 10933-10942.

9 Chidambara Murthy KN, Jayaprakasha GK, Kumar V, Rathore KS, Patil BS. Citrus limonin and its glucoside inhibit colon adenocarcinoma cell proliferation through apoptosis. J Agric Food Chem 2011; 59: 2314-2323.

10 Rahman A, Siddiqui SA, Jakhar R, Kang SC. Growth inhibition of various human cancer cell lines by imperatorin and limonin from poncirus trifoliata rafin. Seeds. Anticancer Agents Med Chem 2014; 15: 236-241.

11 Tanaka T, Kohno H, Tsukio Y, et al. Citrus limonoids obacunone and limonin inhibit azoxymethane-induced colon carcinogenesis in rats. Biofactors 2000; 13: 213-218.

$12 \mathrm{Su}$ LK, Kinzler KW, Vogelstein B, et al. Multiple intestinal neoplasia caused by a mutation in the murine homolog of the APC gene. Science 1992; 256:

\section{Acknowledgments}

This work was supported by Grants-in-Aid for the Third-Term Comprehensive 10-Year Strategy for Cancer Control from the Ministry of Health, Labour, and Welfare of Japan, and also from Grants-in-Aid for Project Future, Relay for Life (Japan Cancer Society), and also supported by the National Cancer Center Research Core Facility (23-A-7).

\section{Abbreviations}

ACF aberrant crypt foci

COX-2 cyclooxygenase-2

DMSO dimethyl sulfoxide

FBS fetal bovine serum

MCP-1 monocyte chemotactic protein-1

PCNA proliferation cell nuclear antigen

PCR polymerase chain reaction

Tcf/Lef T-cell factor/lymphocyte enhancer factor

VCAM-1 vascular cell adhesion molecule-1

\section{Conflict of Interest}

No potential conflicts of interest were disclosed.

$668-670$

13 Oshima M, Oshima H, Kitagawa K, Kobayashi M, Itakura C, Taketo M. Loss of Apc heterozygosity and abnormal tissue building in nascent intestinal polyps in mice carrying a truncated Apc gene. Proc Natl Acad Sci U S A 1995; 92: 4482-4486.

14 Cadigan KM, Nusse R. Wnt signaling: a common theme in animal development. Genes Dev 1997; 11: 3286-3305.

15 Ilyas M. Wnt signalling and the mechanistic basis of tumour development. $J$ Pathol 2005; 205: 130-144.

16 Giles RH, van Es JH, Clevers H. Caught up in a Wnt storm: Wnt signaling in cancer. Biochim Biophys Acta 2003; 1653: 1-24.

17 He TC, Sparks AB, Rago C, et al. Identification of c-MYC as a target of the APC pathway. Science 1998; 281: 1509-1512.

18 Tetsu O, McCormick F. Beta-catenin regulates expression of cyclin D1 in colon carcinoma cells. Nature 1999; 398: 422-426.

19 Shtutman M, Zhurinsky J, Simcha I, et al. The cyclin D1 gene is a target of the beta-catenin/LEF-1 pathway. Proc Natl Acad Sci U S A 1999; 96: 55225527.

20 Mestdagt M, Polette M, Buttice G, et al. Transactivation of MCP-1/CCL2 by beta-catenin/TCF-4 in human breast cancer cells. Int J Cancer 2006; 118: 3542 .

21 Sansom OJ, Meniel VS, Muncan V, et al. Myc deletion rescues Apc deficiency in the small intestine. Nature 2007; 446: 676-679.

22 Sansom OJ, Reed KR, van de Wetering M, et al. Cyclin D1 is not an immediate target of beta-catenin following Apc loss in the intestine. $J$ Biol Chem 2005; 280: 28463-28467.

23 Fong $\mathrm{CH}$, Hasegawa S, Herman Z, Ou P. Limonoid glucosides in commercial citrus juices. J Food Sci 1989; 54: 1505-1506.

24 Ozaki Y, Ayano S, Miyake M, Maeda H, Ifuku Y, Hasegawa S. Limonoid glucosides in juices of Satsuma mandarin (Citrus unshin Marc.). Biosci Biotechnol Biochem 1992; 56: 836-837. 